\title{
Operating SIRTF for maximum lifetime
}

Charles R. Lawrence, Peter Eisenhardt, Jan G. Emming, Paul T. Finley, Thomas Nicholas Gautier, et al.

Charles R. Lawrence, Peter Eisenhardt, Jan G. Emming, Paul T. Finley, Thomas Nicholas Gautier, George Helou, Richard A. Hopkins, Gregory B. Johnson, Jocelyn Keene, Johnny H. Kwok, Jeff H. Lee, Stephen J. Nieczkoski, Thomas L. Roellig, Russell B. Schweickart, "Operating SIRTF for maximum lifetime," Proc. SPIE 4850, IR Space Telescopes and Instruments, (5 March 2003); doi: 10.1117/12.461953

Event: Astronomical Telescopes and Instrumentation, 2002, Waikoloa, Hawai'i, United States 


\title{
Operating SIRTF for maximum lifetime
}

\author{
C. R. Lawrence* ${ }^{*}$, P. Eisenhardt ${ }^{a}$, J. Emming $^{b}$, P. T. Finley ${ }^{b}$, \\ T. N. Gautier ${ }^{a}$, G. Helou ${ }^{c}$, R. A. Hopkins ${ }^{b}$, G. B. Johnson ${ }^{b}$, J. Keene ${ }^{a}$, J. \\ H. Kwok ${ }^{a}$, J. H. Lee ${ }^{b}$, S. J. Nieczkoski ${ }^{b}$, T. L. Roellig ${ }^{d}$, R. Schweickart ${ }^{b}$ \\ a Jet Propulsion Laboratory, California Institute of Technology, Pasadena, CA \\ ${ }^{b}$ Ball Aerospace and Technologies Corp., Boulder, CO \\ ${ }^{c}$ California Institute of Technology, Pasadena, CA \\ $d$ NASA Ames Research Center, Mountain View, CA
}

\begin{abstract}
The instruments of the Space Infrared Telescope Facility (SIRTF) are cooled directly by liquid helium, while the optical system is cooled by helium vapor. The greater the power dissipation into the liquid helium, the more vapor is produced, and the colder the telescope. Observations at shorter wavelengths do not require telescope temperatures as low as those required at longer wavelengths. By taking advantage of this, it may be possible to extend the helium and mission lifetime by $10 \%$ or even $20 \%$.
\end{abstract}

Keywords: Cryogenics, infrared telescopes

\section{INTRODUCTION}

The Space Infrared Telescope Facility ${ }^{1-3}$ will observe from $3.5 \mu \mathrm{m}$ to $160 \mu \mathrm{m}$ with cryogenically-cooled instruments and optics. The nominal mission will end when the liquid helium cryogen runs out. SIRTF was designed to have a lifetime of at least $2.5 \mathrm{yr}$ with the telescope held at $5.5 \mathrm{~K}$ throughout. The current best estimate of lifetime is $5.0 \mathrm{yr}$ with the telescope at $5.5 \mathrm{~K}$. In this paper, we discuss how the lifetime of SIRTF can be extended by letting the telescope temperature fluctuate.

The SIRTF thermal system is shown schematically in Figure 1 (see reference 2 for details), and comprises a telescope mounted to a liquid helium cryostat containing three instruments (IRAC, IRS, and MIPS), a spacecraft bus, a solar array, and a number of components that thermally isolate the cold parts from the warm parts. The cryostat is small, only $360 \mathrm{l}$. Compared to previous cryogenic space missions (e.g., IRAS, COBE, ISO), SIRTF's rate of helium vaporization is expected to be roughly an order of magnitude lower. The cryostat is carefully designed ${ }^{2}$, but SIRTF's outstanding thermal performance is not the result of any magic in the cryostat itself. Rather, it is the result of a thermally favorable orbit combined with agressive use of radiative cooling and thermal isolation of the cold components from the warm comonents. The level of parasitic heat reaching the liquid helium during normal instrument operations is small compared to the active heat load generated by any of the instruments.

The instruments are cooled by a high-conductivity strap connected to the liquid helium tank. The telescope, by contrast, is cooled by helium vapor venting from the cryostat. Two familiar properties of helium are important:

- The vapor pressure of liquid helium varies monotonically from roughly 0.1 torr at $1 \mathrm{~K}$, to 23.5 torr at $2 \mathrm{~K}$. The warmer the liquid in the cryostat, the more helium vapor will be produced.

- The heat of vaporization of helium is $22.6 \mathrm{~J} \mathrm{~g}^{-1}$ at $1.5 \mathrm{~K}$, while the enthalpy of helium at a few kelvin is $5.2 \mathrm{~J} \mathrm{~g}^{-1} \mathrm{~K}^{-1}$. Thus is takes about the same amount of heat to raise the temperature of helium vapor from

* charles.lawrence@jpl.nasa.gov; 818642 1784; M/S 169-327, JPL, 4800 Oak Grove Drive, Pasadena, CA 91109 


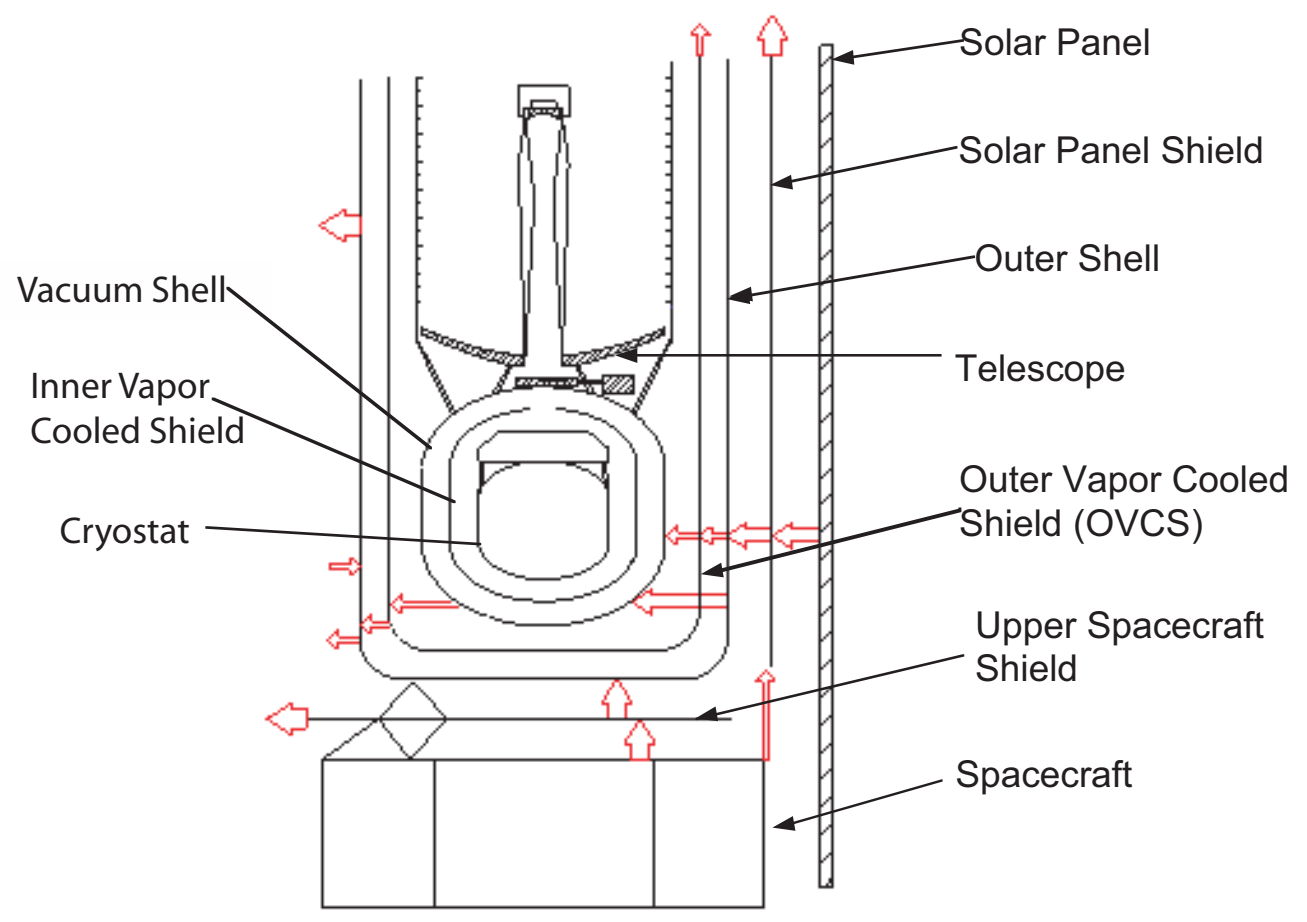

FIG 1.- Schematic of the main thermal components of SIRTF. Extensive measures have been taken to thermally isolate the warm and cold parts from each other, and to take advantage of the nearly $4 \pi$ cold space radiative environment in heliocentric orbit. As a result, the level of parasitic heat reaching the liquid helium during normal instrument operations is small compared to the active heat load generated by the any of the instruments.

$1.5 \mathrm{~K}$ to $5.5 \mathrm{~K}$ as it takes to vaporize it in the first place. This means that helium vapor has as much cooling capacity going from $1.5 \mathrm{~K}$ to $5.5 \mathrm{~K}$ as does helium liquid vaporizing at $1.5 \mathrm{~K}$. It also means that the amount of cooling available to the telescope from venting gas is roughly equal to the active+parasitic heat load on the helium liquid in the tank.

In the following sections, we discuss how these basic characteristics of the SIRTF thermal system can be exploited to maximize the lifetime of the mission.

\section{TEMPERATURE REQUIREMENTS \& EQUILIBRIUM TEMPERATURES}

Table 1 lists the detector and telescope temperatures required by the three instruments. The telescope temperature requirements are set so that thermal emission from the optical system will degrade the overall sensitivity by $\leq 5 \%$ in the given wavelength range. Table 2 and Figure 2 show model calculations of equilibrium telescope temperature as a function of active power dissipation into the cryostat. There are two sources of active power dissipation. The first is the instruments themselves. Based on measurements made during cryogenic testing at Ball Aerospace, IRAC dissipates $4.14 \mathrm{~mW}$, MIPS dissipates $2.71 \mathrm{~mW}$ (with a $\pm 0.3 \mathrm{~mW}$ range depending on the anneal cycle required to be determined in flight), and IRS dissipates $2.26 \mathrm{~mW}$. The second source of active power dissipation into the helium is an electric resistance heater called the "makeup" heater, whose power output can be varied by command between 0 and $25 \mathrm{~mW}$.

Two thermal models, referred to as nominal and worst case, are used. The nominal model uses the bestestimate values for thermal conductances, emissivities, contact resistances, etc., and incorporates data from 
TABLE 1

TEMPERATURE AND REQUIREMENTS

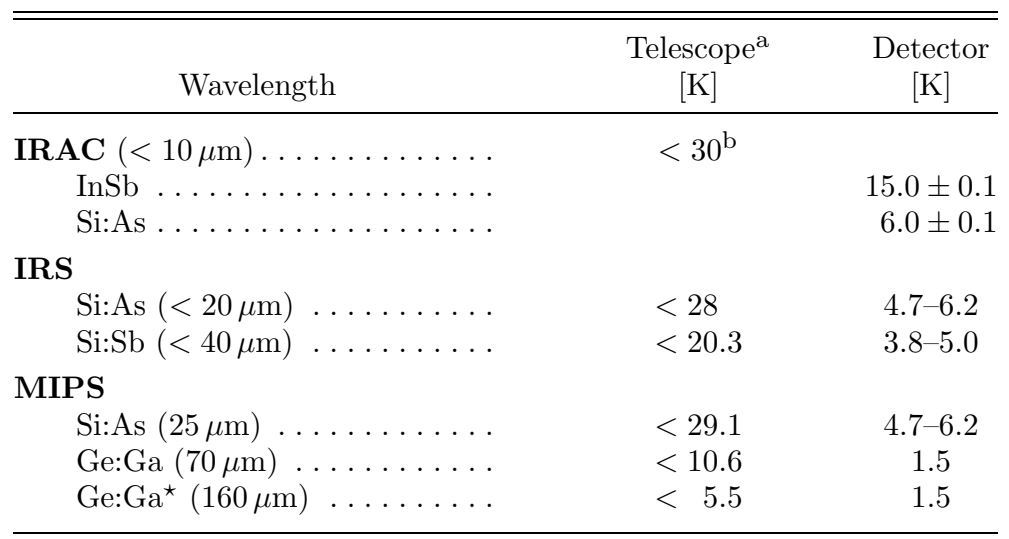

a The primary and secondary mirrors and the secondary support tower are essentially isothermal. The telescope temperatures in the table give $\leq 5 \%$ degradation of sensitivity.

TABLE 2

Equilibrium Temperatures By Power Dissipation

\begin{tabular}{|c|c|c|c|c|c|c|}
\hline \multirow[b]{2}{*}{$\begin{array}{l}\text { ACTIVE HEAT LOAD } \\
{[\mathrm{mW}]}\end{array}$} & \multicolumn{3}{|c|}{ NOMINAL ${ }^{\mathrm{a}}$ MODEL } & \multicolumn{3}{|c|}{ WORST-CASE ${ }^{\mathrm{a}}$ MODEL } \\
\hline & $\begin{array}{c}\text { Parasitic load } \\
{[\mathrm{mW}]}\end{array}$ & $\begin{array}{c}T_{\text {bath }} \\
{[\mathrm{K}]}\end{array}$ & $\begin{array}{c}T_{\text {telescope }} \\
{[\mathrm{K}]}\end{array}$ & Parasitic load ${ }^{\mathrm{c}}$ & $T_{\text {bath }}$ & $T_{\text {telescope }}$ \\
\hline$\ldots \ldots$ & 1.92 & 1.18 & 14.7 & 2.61 & 1.22 & 18.4 \\
\hline$\ldots \ldots \ldots \ldots$ & 1.34 & 1.19 & 13.5 & 2.01 & 1.23 & 17.4 \\
\hline$\ldots \ldots \ldots$ & 0.87 & 1.20 & 11.8 & 1.47 & 1.24 & 16.2 \\
\hline 2.26 (IRS) & & & 11.4 & & & 15.9 \\
\hline 2.71 (MIPS) . . & & & 10.3 & & & 15.2 \\
\hline $3.00 \ldots \ldots \ldots \ldots$ & 0.54 & 1.22 & 9.6 & 1.01 & 1.25 & 14.7 \\
\hline $4.00 \ldots \ldots \ldots \ldots \ldots$ & 0.34 & 1.23 & 7.2 & 0.69 & 1.26 & 12.9 \\
\hline $4.14($ IRAC $) \ldots$. & & & 7.6 & & & 13.1 \\
\hline $4.85 \ldots \ldots$ & 0.33 & 1.24 & 5.5 & 0.50 & 1.26 & 11.1 \\
\hline$\ldots \ldots \ldots$ & 0.33 & 1.24 & 5.3 & 0.48 & 1.27 & 10.8 \\
\hline 6.00 & 0.31 & 1.25 & 4.1 & 0.38 & 1.28 & 8.8 \\
\hline 7.00 & 0.31 & 1.26 & 3.4 & 0.35 & 1.28 & 7.2 \\
\hline$\ldots \ldots$ & 0.30 & 1.27 & 2.9 & 0.33 & 1.29 & 6.0 \\
\hline 8.59 & 0.30 & 1.27 & 2.9 & 0.32 & 1.30 & 5.5 \\
\hline$\ldots \ldots \ldots$ & 0.30 & 1.28 & 2.7 & 0.32 & 1.30 & 5.2 \\
\hline
\end{tabular}

a Nominal and worst-case refer to the two thermal models used by Ball and the assumptions on which they are based.

b Active heat load is the sum of instrument power dissipation and the makeup heater, which is adjustable by command.

${ }^{\mathrm{c}}$ Includes the equivalent of $0.30 \mathrm{~mW}$ attributed to film flow across the porous plug.

cryogenic tests of the entire cold telescope assembly at Ball Aerospace in late 2001. The worst case model uses values for thermal conductances, emissivities, etc., that are at the extreme unfavorable end of the uncertainty ranges for each, combined not statistically, but assuming that extreme values are achieved simultaneously.

Essential facts about the SIRTF thermal system can be seen from Tables 1 and 2 and Figure 2:

- The more power dissipated in the helium, the lower the equilibrium telescope temperature.

- None of the three instruments dissipates enough power by itself to maintain a telescope temperature of $5.5 \mathrm{~K}$. 


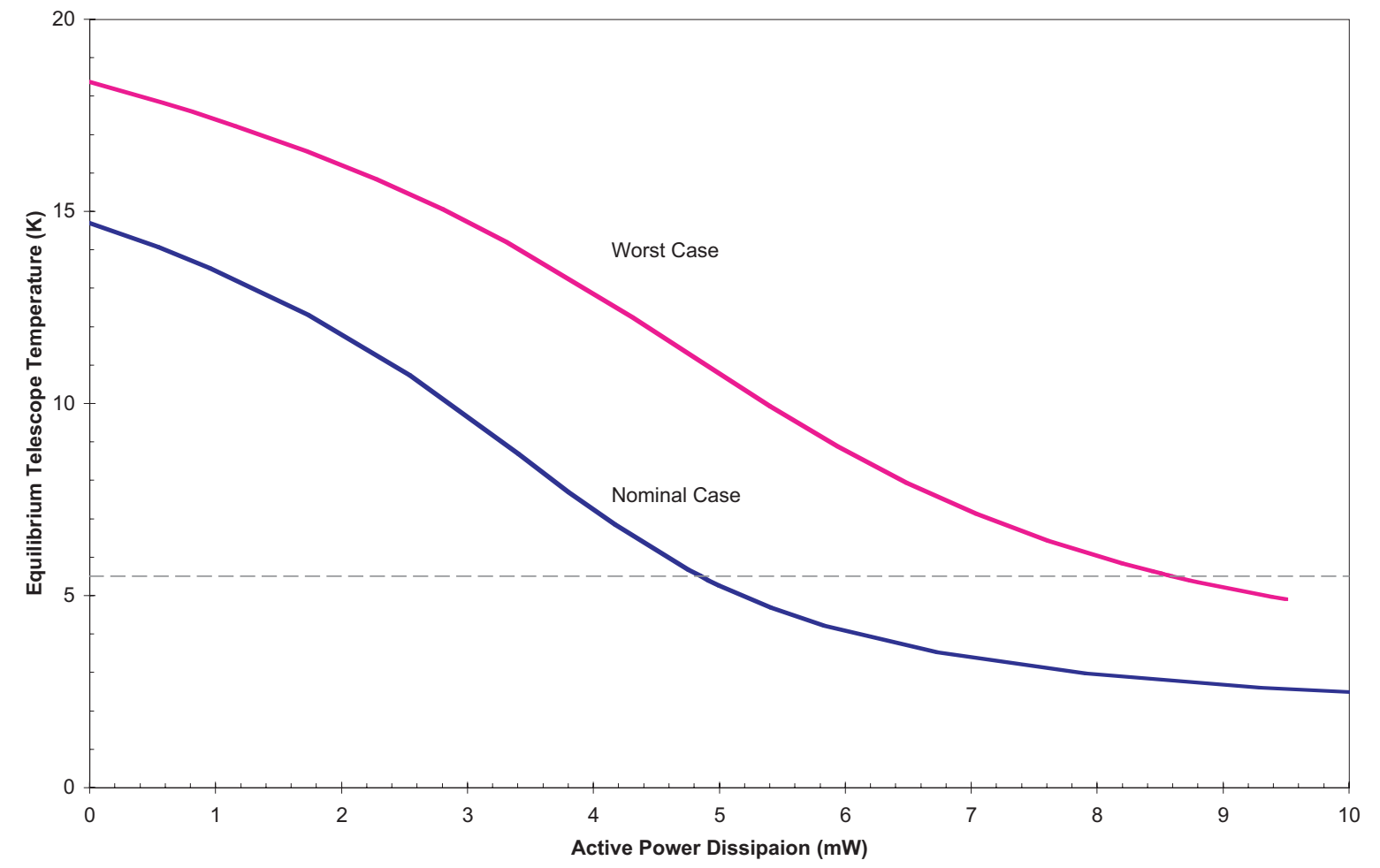

FIG 2.- Telescope temperature as a function of power dissipation into the helium (see also Table 2). The two curves give results both for the best-estimate thermal model ("nominal") and for a "worst-case" model in which emissivities and conductances are assumed to take on extreme values simultaneously. The $5.5 \mathrm{~K}$ telescope temperature required for $160 \mu \mathrm{m}$ observations is achieved with active heat input (instrument + makeup heater) of $4.85 \mathrm{~mW}$ (nominal case) or $8.59 \mathrm{~mW}$ (worst case).

- In both nominal and worst cases, IRS and IRAC dissipate enough power to maintain their required telescope temperatures of $20.3 \mathrm{~K}$ and $30 \mathrm{~K}$, respectively.

- MIPS dissipates enough power to maintain the required telescope temperature for $25 \mu \mathrm{m}$ in both nominal and worst cases, and for $70 \mu \mathrm{m}$ in the nominal case. For $160 \mu \mathrm{m}$ observations (both cases) and $70 \mu \mathrm{m}$ observations (worst case), additional power must be dissipated in the helium by the makeup heater.

To maintain a constant telescope temperature of $5.5 \mathrm{~K}$, the makeup heater would have to supply $(4.85-$ 4.14) $\mathrm{mW}$ for IRAC, $(4.85-2.71) \mathrm{mW}$ for MIPS, and $(4.85-2.26) \mathrm{mW}$ for IRS. The modelled helium bath temperature corresponding to a $5.5 \mathrm{~K}$ telescope is $1.2375 \mathrm{~K}$ (given to four decimals not because it is known to that accuracy, but for comparison with the figures that follow). As indicated in $\S 1$, the lower the helium temperature the less helium vapor is produced. Since most observations do not require a $5.5 \mathrm{~K}$ telescope, helium can be saved by not dissipating additional power with the makeup heater when it is not needed, thereby letting the helium cool below $1.2375 \mathrm{~K}$ and the telescope warm above $5.5 \mathrm{~K}$.

To show how this might work in practice, we first discuss transient effects in the thermal system. For simplicity, we will discuss transient behavior only in the nominal case.

\section{TRANSIENT BEHAVIOR}

Suppose that SIRTF has reached thermal equilibrium with IRS operating and the makeup heater supplying an additional $2.59 \mathrm{~mW}$, for a total dissipated power into the helium of $4.85 \mathrm{~mW}$. According to Table 2, the telescope temperature will be $5.5 \mathrm{~K}$. If the makeup heater is turned off, the total dissipated power will drop to 
$2.26 \mathrm{~mW}$. The helium bath temperature will start to drop, and the helium vapor pressure and the flow rate of helium vapor out of the cryostat will drop correspondingly. The telescope temperature will rise, maintaining the balance between parasitic heat on the telescope and the cooling capacity of the helium vapor venting from the cryostat.

Figure 3 shows the telescope and helium bath temperatures as a function of time for this situation, as well as the reverse situation when the dissipated power increases from $2.26 \mathrm{~mW}$ to $4.85 \mathrm{~mW}$ at $t=0$. Calculations are shown for both the beginning of the mission, with the cryostat $73 \%$ full (left-hand panels), and near the end of the mission, with the cryostat only $10 \%$ full (right-hand panels).

The most important lesson from Figure 3 is that it takes a long time to for the telescope temperature to reach equilibrium, roughly two weeks at the beginning of the mission, and one week near the end.

It can be seen also in Figure 3 that the bath temperature overshoots its equilibrium value. This is due primarily to the fact that the pressure drop in the vent tube depends on the vent tube temperature distribution, which changes with the telescope temperature. For example, in the $4.85 \rightarrow 2.26 \mathrm{~mW}$ case at the beginning of the mission (bottom left panel), when the power is reduced at $t=0$ the helium bath temperature drops rapidly at first, but levels out after about six days. As the temperatures of the telescope and outer end of the cryostat vent tube continue to rise, however, the pressure drop in the vent tube increases. The vapor pressure inside the cryostat increases, and the helium temperature rises slowly to its equilibrium value.

\section{OPERATIONAL SCENARIOS AND LIFETIME}

Suppose that the telescope temperature were allowed to rise during IRS and IRAC observations, when the direct instrument power dissipation is significantly less than $4.85 \mathrm{~mW}$. Extra heat would need to be added before MIPS observations begin $\dagger$ to bring the telescope temperature down to $5.5 \mathrm{~K}$; however, for much of the time IRS and IRAC were on, the direct heat input into the helium would be less than $4.85 \mathrm{~mW}$, resulting in a net savings of helium.

Minimum helium use requires keeping the temperature of the helium as low as possible for as long as possible (minimizing the time integral of the helium bath temperature), and then raising it as quickly as possible to $1.2375 \mathrm{~K}$. Thus the ideal shape of a plot of helium bath temperature vs. time is a step function, with the temperature before the step the IRS or IRAC equilibrium temperature and the temperature after the step $1.2375 \mathrm{~K}$. The step is achieved by adding heat $\Delta Q=\int_{T_{1}}^{1.2375 \mathrm{~K}} m_{\mathrm{He}} c d T$, where $c$ is the heat capacity of helium, in a "pulse" of high power and short duration. The pulse ends when the helium reaches $1.2375 \mathrm{~K}$, after which the total active heat input into the helium is maintained at $4.85 \mathrm{~mW}$ by suitable adjustment of the makeup heater. Over the next few days the telescope temperature gradually drops to $5.5 \mathrm{~K}$. The heat pulse is timed during the middle of the IRS or IRAC run so that $5.5 \mathrm{~K}$ has been reached when MIPS is switched on.

Figure 4 shows three examples. Instruments are operated in the sequence IRS-IRAC-MIPS for 3, 7, and 14 days each in the top, middle, and bottom panels, respectively. No makeup heat is added during the IRS run. During the IRAC run, several days before the MIPS run will start, the makeup heater is adjusted to dissipate $25 \mathrm{~mW}$, and left on until the helium temperature increases the correct amount. The makeup heater is then adjusted to give a total power dissipation of $4.85 \mathrm{~mW}$. The additional vapor venting from the cryostat because of the warmer helium then gradually brings the telescope temperature down to $5.5 \mathrm{~K}$ at the start of the MIPS run.

For the $3 \times 3=9$ day and $3 \times 7=21$ day cycles, it turns out that the bath temperature profile that minimizes helium use (i.e., a step function up to $1.2375 \mathrm{~K}$ ) would produce too little helium vapor to cool the telescope to $5.5 \mathrm{~K}$ in the time available before the MIPS run begins. A larger than "ideal" heat pulse is required to raise the bath temperature above $1.2375 \mathrm{~K}$ for a time. The extra vapor produced cools the telescope more quickly. This is not necessary in the $3 \times 14=42$ day cycle, which as a result is more efficient in helium utilization.

$\dagger$ This ensures that $160 \mu \mathrm{m}$ observations can be made at the beginning of a MIPS run. 

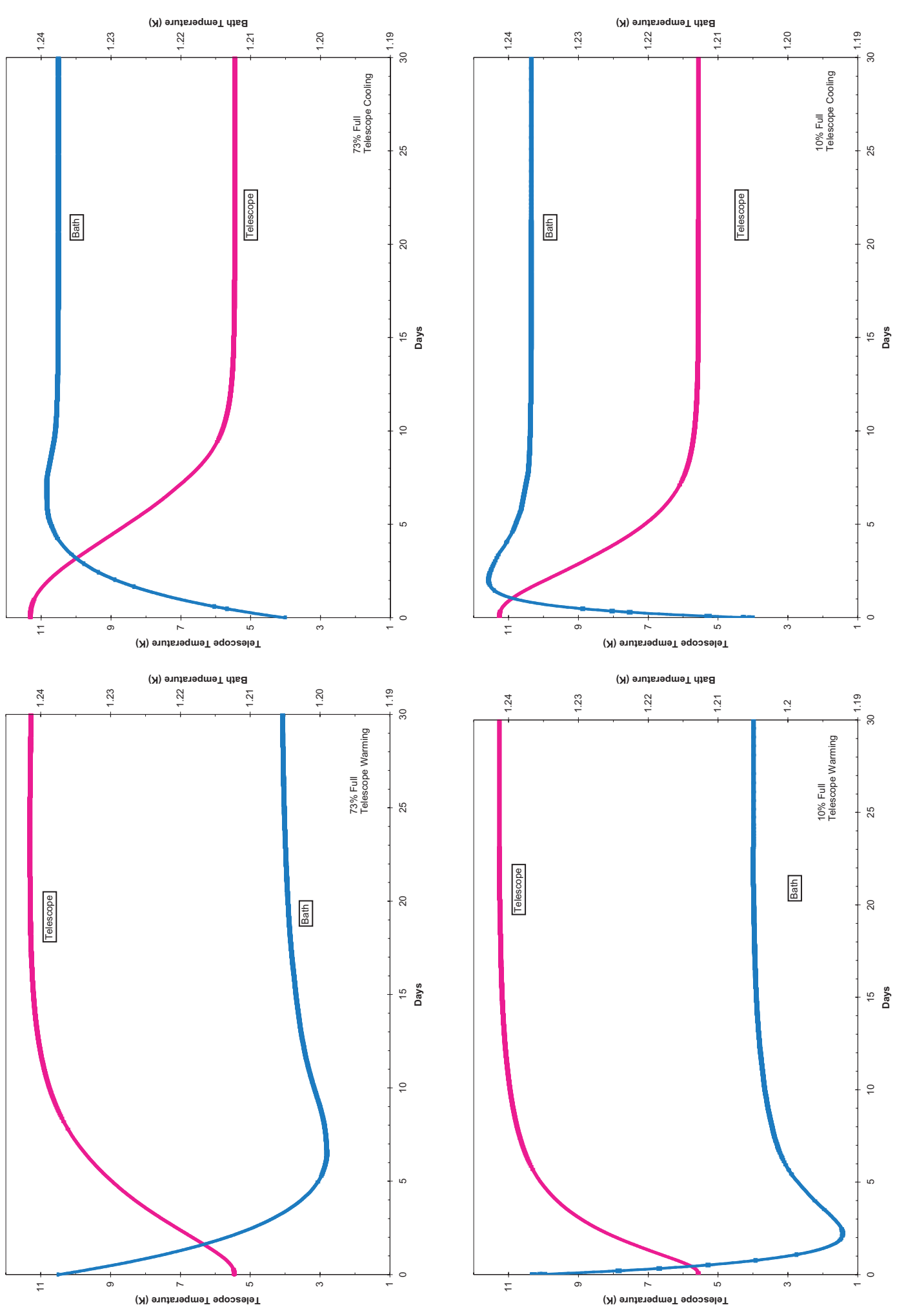

FIG 3.- Telescope and helium bath temperatures as a function of time after a sudden change in active heat input into the helium. The four panels show two cases $-4.85 \mathrm{~mW} \rightarrow 2.26 \mathrm{~mW}$ (bottom panels), and $2.26 \mathrm{~mW} \rightarrow 4.85 \mathrm{~mW}$ (top panels) - at two different times, once early in the mission, with the cryostat $73 \%$ full (left-hand panels), and once near the end of the mission, with the cryostat only $10 \%$ full (right-hand panels). In all panels, at $t=0$ the system is in equilibrium. The active heat input into the helium is the sum of the instrument dissipation and the makeup heater dissipation. 

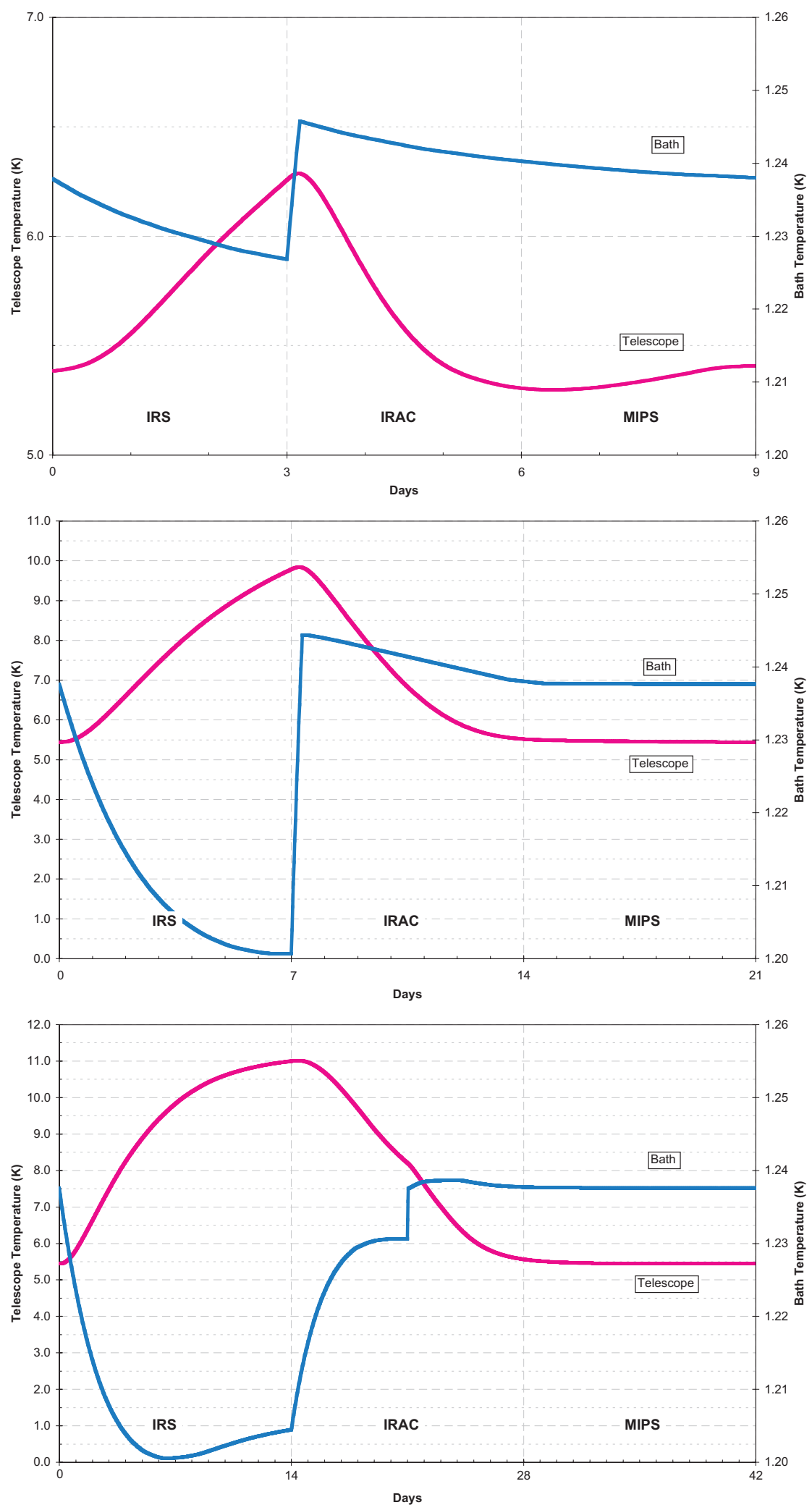

FIG 4.- Telescope and bath temperature as a function of time for three operational scenarios. IRS, IRAC, and MIPS are operated in sequence for 3, 7, or 14 days each (top, middle, and bottom panels, respectively). 
At the end of the mission, with the helium nearly gone, there is no need for the extra heat pulse. The helium bath temperature changes quickly enough when the internal dissipation jumps to $4.85 \mathrm{~mW}$ that the telescope temperature curves are essentially indistinguishable from those in Figure 4. Over the course of the mission, then, the length of the heat pulse required to cool the telescope for MIPS observations would decrease approximately linearly from its initial value to zero.

Figure 5 shows SIRTF's helium lifetime estimate as a function of cycle length for operational scenarios like those in Figure 4. Cycle length zero corresponds to keeping the telescope at $5.5 \mathrm{~K}$ always, which results in a 5.03 year lifetime. For the 42 -day cycle, the lifetime estimate is 6.11 years, a $20 \%$ increase.

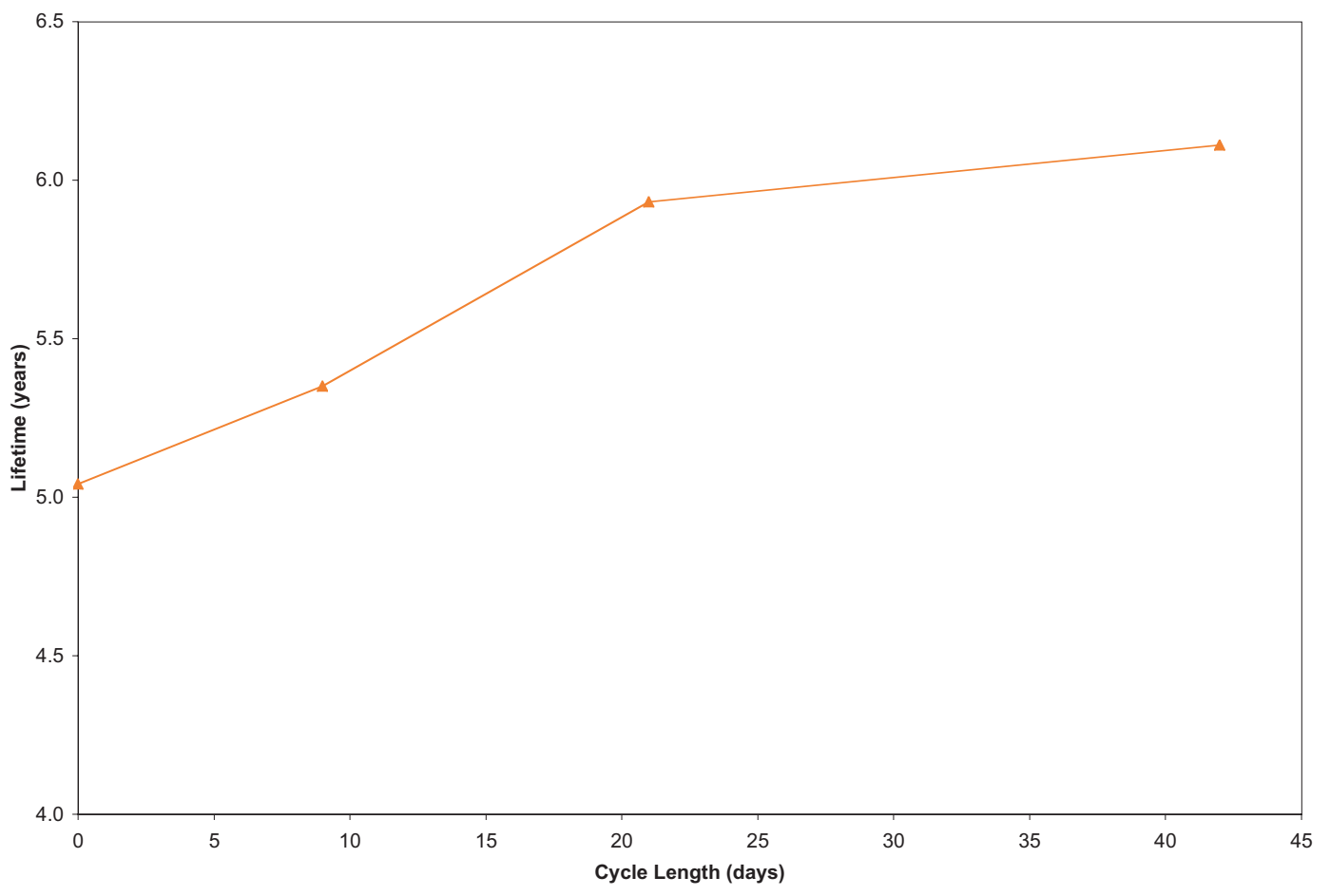

FIG 5.- Helium lifetime estimate as a function of cycle length for scenarios like those of Figure 4 . The longer the cycle time the greater the lifetime. Most of the potential gains are realized when each instrument is operated for about a week (21 day cycle time).

Figure 6 shows what happens if the instrument order is changed to IRAC-IRS-MIPS in a 21-day cycle. Rather than the helium lifetime estimate of 5.93 years produced by the 21-day cycle in Figure 4, this "bad" scenario results in a helium lifetime estimate of only 5.5 years.

\section{SUMMARY}

The foregoing examples illustrate the potential for extending the helium lifetime of SIRTF by taking advantage of the fact that different instruments require different telescope temperatures. Several points deserve emphasis:

- The calculations presented here are based on a thermal model that has been developed over several years at Ball Aerospace, and that has been verified to the extent possible during cryogenic testing at Ball. Nevertheless, the true thermal behavior of SIRTF will not be known until flight. All plans for thermal operation must be re-evaluated after launch, when flight data are available.

- There are many other constraints on the instrument schedule and cycle time than just helium utilization. Long, 


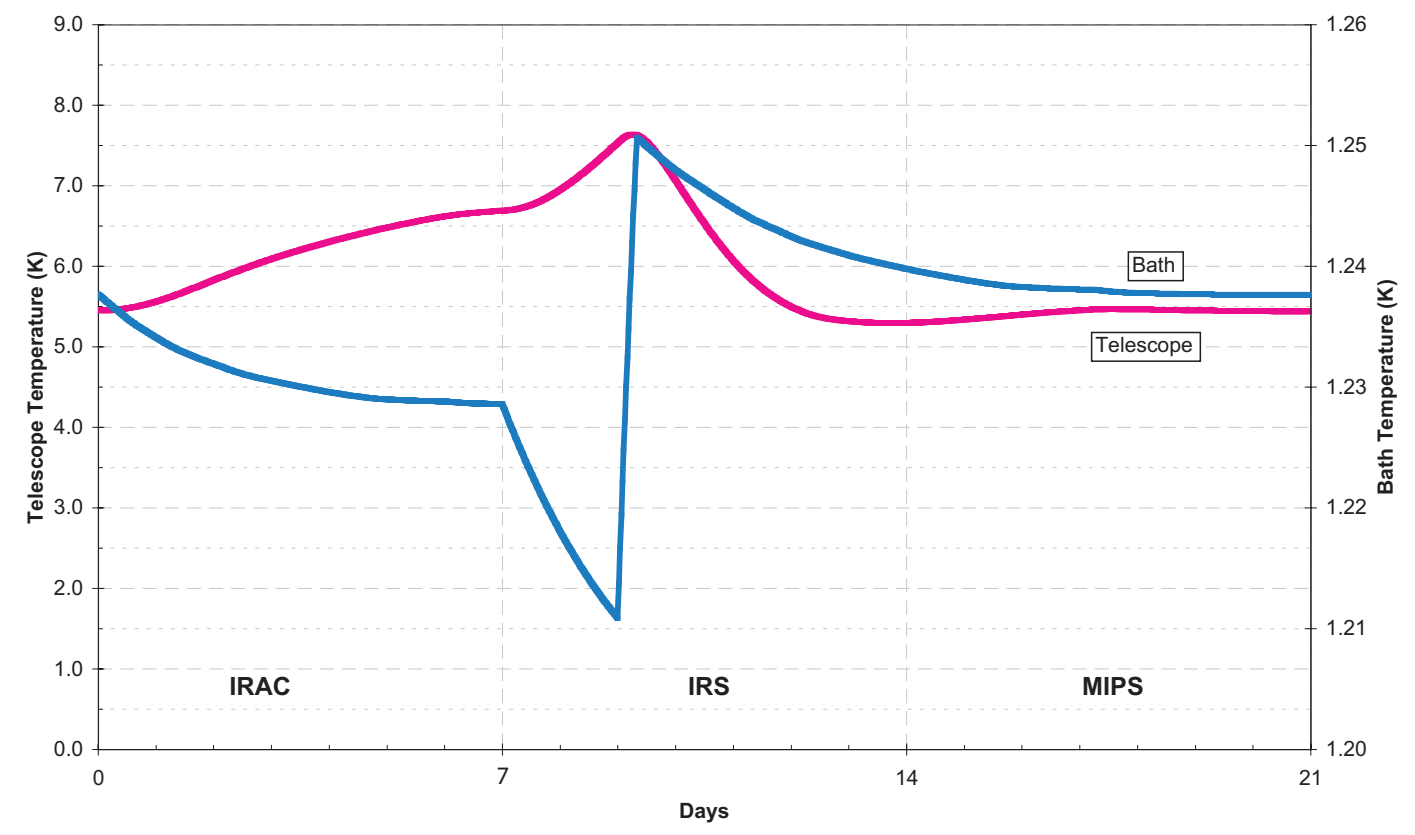

FIG 6.-Telescope and bath temperature as a function of time for an operational scenario like the middle panel of Figure 4, but with the order of instruments changed to IRAC, IRS, and MIPS. This order provides little helium savings.

regular instrument cycles will not always be possible in a real observing schedule, especially during the first year.

- Many variants on the operational scenarios given here are possible, including some that would be much more agressive in conserving helium. For example, if $160 \mu \mathrm{m}$ observations were collected into $160 \mu \mathrm{m}$-only periods, the telescope temperature could be kept above $5.5 \mathrm{~K}$ for a large fraction of the time. There are drawbacks to such schemes in terms of observational efficiency and potential science loss; however, the availability of more agressive operational scenarios provides considerable robustness to the mission. Even if on-orbit thermal performance falls short of expectations, a long lifetime can be achieved.

\section{REFERENCES}

1. D. B. Gallagher, W.. R. Irace, and M. W. Werner. "SIRTF mission," in IR Space Telescopes and Instruments, this volume.

2. R. A. Hopkins, R. B. Schweickart, and S. M. Volz, "Cryogenic/thermal system for the SIRTF cryogenic telescope assembly," in IR Space Telescopes and Instruments, this volume.

3. P. T. Finley, R. L. Oonk, and R. B. Schweickart, "Thermal performance verification of the SIRTF cryogenic telescope assembly," in IR Space Telescopes and Instruments, this volume. 